\title{
Les Plaies Pénétrantes De L'abdomen Par Armes À Feu : Aspects Épidémiologiques, Cliniques, Lésionnels Et Thérapeutiques Au CHU Communautaire De Bangui, Centrafrique
}

\author{
Pierre Alfred Issa Mapouka, \\ Service de traumatologie et d'orthopédie, \\ Centre Hospitalier Universitaire Communautaire, Bangui \\ Valère Ndoma Ngatchoukpo, \\ Service de chirurgie pédiatrique, Centre Hospitalier Universitaire \\ Pétula Anicette Ngboko Mirotiga, \\ Don Rodrigue Nabia, \\ Vianney Auxence Roméo Fioboy, \\ Bertrand Jean de Dieu Tékpa, \\ Service de traumatologie et d'orthopédie, \\ Centre Hospitalier Universitaire Communautaire, Bangui
}

Doi:10.19044/esj.2019.v15n36p475 URL:http://dx.doi.org/10.19044/esj.2019.v15n36p475

Résumé

Objectif : Décrire les aspects épidémiologiques, cliniques, lésionnels et thérapeutiques des plaies pénétrantes de l'abdomen par armes à feu dans un contexte de conflit armé. Patients et méthodes : Il s'agit d'une étude rétrospective descriptive portant sur les plaies pénétrantes de l'abdomen par armes à feu. Etaient inclus, tous les patients traités dans le service de traumatologie et d'orthopédie du Centre Hospitalier Universitaire Communautaire de Bangui du $1^{\text {er }}$ Octobre 2013 au 31 Mars 2014. Résultats : La série comportait 56 patients : 52 hommes $(92,8 \%)$ et 4 femmes $(7,2 \%)$. L'âge moyen des patients était de 29,8 $\pm 3,9$ ans. Les belligérants $(39,3 \%)$ étaient plus touchés suivis des élèves et étudiants $(21,4 \%)$. Les circonstances des plaies étaient les affrontements $(48,2 \%)$, les agressions et rixes $(30,4 \%)$ et les balles perdues $(21,4 \%)$. Les moyens de transport des patients étaient les véhicules privés $(44,6 \%)$, les ambulances non médicalisées $(35,8 \%)$, les motos-taxis $(12,5 \%)$ et les pousses-pousses $(7,1 \%)$. Le délai moyen d'admission était de 2,5 heures. Les signes cliniques étaient : la douleur abdominale (100\%), l'irritation péritonéale $(76,8 \%)$, le choc hémorragique (23,2\%), l'éviscération $(14,3 \%)$ et l'écoulement du liquide digestif $(9 \%)$. La laparotomie exploratrice était réalisée chez 51 patients (91\%) tandis que 5 
patients (9\%) ont fait l'objet d'une abstention chirurgicale. Des lésions d'organes étaient retrouvées dans $88,2 \%$ des cas. Les organes plus atteints étaient le grêle $(39,2 \%)$, le colon $(23,7 \%)$ et le foie $(9,3 \%)$. Les lésions concernaient un organe dans $51,1 \%$ des cas et pluriviscérales dans $48,9 \%$ des cas. Les principaux gestes chirurgicaux pratiqués étaient l'excision-suture des organes ( 25 cas), les stomies ( 21 cas) et la résection-anastomose (17 cas). La morbidité était de $40 \%$ dominée par la suppuration pariétale $(56 \%)$. La mortalité était de 17,9\%. La durée moyenne d'hospitalisation était de 10 jours. Conclusion : Les plaies abdominales par armes à feu sont fréquentes en période de conflit armé. Elles touchent les sujets jeunes majoritairement les hommes. Les lésions organiques sont souvent multiples. Dans notre contexte, la prise en charge préhospitalière était inexistante et insuffisante en milieu hospitalier. Ce qui était à l'origine d'une morbidité et mortalité élevées.

Mots clés: Abdomen, Plaies pénétrantes, Arme à feu, Traitement, Centrafrique 


\title{
Penetrating abdominal wounds by firearm: epidemiological clinical and therapeutic aspects at Bangui Community Teaching Hospital, Central African Republic
}

\author{
Pierre Alfred Issa Mapouka, \\ Service de traumatologie et d'orthopédie, \\ Centre Hospitalier Universitaire Communautaire, Bangui \\ Valère Ndoma Ngatchoukpo, \\ Service de chirurgie pédiatrique, Centre Hospitalier Universitaire \\ Pétula Anicette Ngboko Mirotiga, \\ Don Rodrigue Nabia, \\ Vianney Auxence Roméo Fioboy, \\ Bertrand Jean de Dieu Tékpa, \\ Service de traumatologie et d'orthopédie, \\ Centre Hospitalier Universitaire Communautaire, Bangui
}

\begin{abstract}
Objective: Describe the epidemiological, clinical, and lesional aspects and to report the results of the management of penetrating abdominal wounds by firearms in a context of armed conflict. Patients and methods: This is a retrospective descriptive study of penetrating abdominal wounds by firearms. Included were all patients treated and followed in the Trauma and Orthopedic Department of the Bangui Community University Hospital Center from October 1, 2013 to March 31, 2014. Results: The series included 56 patients: 52 men $(92.8 \%)$ and 4 women $(7.2 \%)$. The average age of the patients was $29.8 \pm 3,9$ years old. The means of transport for the patients were private vehicles $(44.6 \%)$, non-medical ambulances $(35.8 \%)$, motorcycle taxis $(12.5 \%)$ and pushpushes $(7.1 \%)$. The average admission time was 2.5 hours. The circumstances of the wounds were clashes (48.2\%), assaults (30.4\%) and stray bullets $(21.4 \%)$. The clinical signs were: abdominal pain (100\%), peritoneal irritation (76.8\%), hemorrhagic shock (23.2\%), evisceration $(14.3 \%)$ and the flow of digestive fluid (9\%). Exploratory laparotomy was performed in 51 patients and 5 patients underwent surgical abstention. Intra-abdominal organ lesions were found in 45 patients $(88.2 \%)$ while in 6 patients $(11.8 \%)$, the laparotomy was white. The most affected organs were hail (39.2\%), colon (23.7\%) and liver $(9.3 \%)$. The wounds concerned a single organ in 22 patients
\end{abstract}


(48.9\%) whereas in 23 patients $(51.1 \%)$ they were plurivisceral. Morbidity was $40 \%$ and mortality was $17,9 \%$. The average hospital stay was 10 days. Conclusion: Abdominal gunshot wounds affect young people, particularly men in times of armed conflict. Organic lesions are often multiple. Prehospital care is non-existent and insufficient in a hospital setting which causes high morbidity and mortality.

Keywords: Abdomen, Penetrating wounds, Firearm, Treatment, Central African Republic

\section{Introduction}

Les plaies de l'abdomen sont des traumatismes avec rupture de la continuité pariétale abdominale. Si ces plaies atteignent la cavité péritonéale, on parle de plaies pénétrantes ; lors qu'elles entrainent une lésion des viscères sous-jacents, la plaie est dite perforante (Dieng, 2002). Les plaies pénétrantes par armes à feu s'accompagnent dans $87 \%$ des cas de lésions viscérales intraabdominales (Moore, 1985). La prolifération des armes légères qui suivent les guerres civiles et les changements armés internes du processus politique dans la sous-région a augmenté le taux de criminalité ainsi que l'incidence des plaies pénétrantes de l'abdomen (Adesanya, 1998). Au plan thérapeutique, la laparotomie en urgence reste indiquée compte tenu de la fréquence et de la multiplicité des lésions viscérales observées (Monneuse, 2004). Cette étude a pour but de décrire les aspects épidémiologiques, cliniques, lésionnels et de rapporter le résultat de la prise en charge des plaies pénétrantes de l'abdomen par armes à feu dans un contexte de conflit armé.

\section{Patients et méthodes}

Il s'agit d'une étude rétrospective descriptive portant sur les plaies pénétrantes de l'abdomen par armes à feu chez des patients pris en charge au service de traumatologie du Centre Hospitalier Universitaire Communautaire de Bangui durant le conflit armé en Centrafrique sur la période du $1{ }^{\mathrm{er}}$ Octobre 2013 au 31 Mars 2014 soit 6 mois. Le recueil des données était fait à partir des registres des urgences chirurgicales, du bloc opératoire et des dossiers d'hospitalisation. Etaient inclus dans l'étude tous les patients traités et suivis après leur sortie et ayant un dossier complet. Les patients victimes d'une plaie pénétrante de l'abdomen par arme blanche et des traumatismes fermés et ceux ayant un dossier incomplet étaient exclus. Les paramètres étudiés étaient : l'âge, le sexe, le profil des victimes, la provenance, les circonstances de survenue, les moyens de transport, le délai d'admission, le bilan clinique et para clinique, les gestes chirurgicaux pratiqués, la morbidité, la mortalité et la durée d'hospitalisation. Les données recueillies sur une fiche d'enquête étaient traitées à l'aide des logiciels Epi-info 2012. 


\section{Résultats}

\section{Aspect épidémiologique}

Durant la période de l'étude, 1370 patients étaient reçus au service des urgences chirurgicales dont 370 pour un traumatisme abdominal parmi lesquels 74 avaient une plaie pénétrante de l'abdomen par arme à feu, soit $5,4 \%$ des admissions et $20 \%$ des traumatismes abdominaux. Cinquante et six (56) patients étaient retenus et 18 exclus : 7 patients décédés à l'arrivée, 2 décès pendant la réanimation préopératoire et 9 patients dont les dossiers étaient incomplets. Le plus grand nombre de patients était reçu au mois de Décembre 2013 avec 32 patients (57,1\%). L'âge moyen était de 29,84 $\pm 3,9$ ans (extrêmes 15 ans et 63 ans). La répartition des tranches d'âge est rapportée dans le tableau I. Le sexe masculin avait prédominé avec 52 cas $(92,8 \%)$; le sex ratio est de 13. Le profil des patients était: les groupes armés (belligérants), 22 cas $(39,3 \%)$; les élèves et étudiants, 12 cas $(21,4 \%)$; les militaires, 7 cas $(12,5 \%)$; les commerçants et les cultivateurs avec 4 cas chacun $(7,1 \%)$; les ménagères et les sans emploi, 3 cas chacun $(5,4 \%)$ et un salarié $(1,8 \%)$. Cinquante et un patients $(91 \%)$ provenaient de la capitale Bangui et les cinq autres $(9 \%)$ de l'intérieur du pays. Les moyens de transport étaient: un véhicule privé dans 25 cas $(44,6 \%)$, une ambulance non médicalisée dans 20 cas $(35,8 \%)$, une moto-taxi dans 7 cas $(12,5 \%)$ et un pousse-pousse dans 4 cas (7,1\%). Le délai d'admission au service des urgences chirurgicales allait de 50 minutes à 6 heures chez 40 blessés $(71,4 \%)$, de 7 heures à 24 heures chez 10 blessés $(17,9 \%)$ et au-delà de 24 heures chez 6 blessés (10,7\%). Le délai moyen d'admission était de 2,5 heures (extrêmes : 50 minutes et 72 heures). Les circonstances des plaies étaient : les affrontements, 27 cas $(48,2 \%)$; les agressions et rixes, 17 cas $(30,4 \%)$ et les balles perdues, 12 cas $(21,4 \%)$. Les plaies relevaient d'une balle dans 47 cas (84\%) et des éclats de grenade dans 9 cas $(16 \%)$.

\section{Aspects clinique et para clinique}

Les plaies étaient uniques chez 36 patents $(64,3 \%)$, multiples chez 20 patients $(35,7 \%)$. La région antérieure de l'abdomen est touchée dans $89,3 \%$. La répartition des plaies selon leur siège est présentée dans le tableau II. Les signes cliniques étaient: la douleur abdominale (100\%), le syndrome d'irritation péritonéale (43 cas) (76,7\%), l'état de choc hémorragique (13 cas) (23,2\%), l'éviscération (épiploon, grêle, colon) (8 cas) $(14,3 \%)$ et l'écoulement du liquide digestif (5 cas) (9\%). Les examens biologiques réalisés étaient le groupe sanguin/rhésus chez tous les patients; le taux d'hémoglobine chez 14 patients (25\%) et le taux d'hématocrite chez 12 patients $(21,4 \%$. La radiographie de l'abdomen sans préparation (ASP) était réalisée chez deux patients suspects d'une plaie thoraco-abdominale mais dont les signes cliniques abdominaux n'étaient pas évidents (figure 1). Aucune 
échographie abdominale n'était réalisée car non disponible pendant la période de l'étude.

\section{Aspect lésionnel}

La laparotomie exploratrice était réalisée chez 51 patients $(91 \%)$ tandis que 5 patients $(9 \%)$ étaient surveillés cliniquement après le parage de leur plaie sous anesthésie locale.Au cours de la laparotomie, 97 plaies d'organes intra-abdominaux étaient retrouvées chez 45 patients $(88,2 \%)$; chez les 6 autres $(11,8 \%)$, elle était blanche. Les organes intra-abdominaux les plus lésés étaient le grêle, 38 plaies $(39,2 \%)$, colon, 23 plaies $(23,7 \%)$ et le foie, 9 plaies $(9,3 \%)$. Les plaies concernaient un seul organe chez 23 patients $(51,1 \%)$ et 2 à 4 organes chez 22 patients $(48,9 \%)$.

\section{Aspect thérapeutique}

En préopératoire, la réanimation était nécessaire chez $21(41,1 \%)$ des 51 patients dont l'indication de la laparotomie était posée. Les gestes chirurgicaux pratiqués sont présentés dans le tableau II. Les suites opératoires étaient compliquées chez $25(49 \%)$ des patients opérés. Il s'agissait de 14 cas $(56 \%)$ de suppuration de la paroi, 4 cas $(16 \%)$ de choc hémorragique, 3 cas (12\%) de choc septique, 2 cas $(8 \%)$ de péritonite et 2 cas $(8 \%)$ de fistule digestive. Dix patients $(17,9 \%)$ sont décédés : 5 de choc hémorragique, 3 de septicémie et 2 d'anémie. La durée moyenne d'hospitalisation était de 10 jours avec des extrêmes de 5 heures et 54 jours.

\section{Discussion}

\section{Aspect épidémiologique}

Dans l'étude, les plaies abdominales par armes à feu ont représenté $5,4 \%$ des traumatismes abdominaux admis. Bodalal et al en Libye et de Thiam et al au Mali avaient trouvé dans le même contexte une fréquence respective de 7,60\% et 7,8\% (Bodalal, 2013; Thiam, 2018). L'âge moyen était de

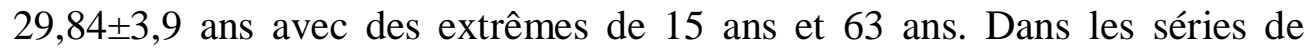
Mnguni et al en Afrique du Sud et Bahebeck et al au Cameroun en pratique civile, cette moyenne d'âge était de 29,2 ans et 32 ans avec des extrêmes de 12 ans et 70 ans et de 10 ans à 63 ans (Mnguni, 2012 ; Bahebeck,2005). Les victimes hommes étaient plus touchées avec 92,8\% dans cette étude. Thiam avait également trouvé dans sa série une prédominance masculine de $96 \%$ (Thiam, 2018). Ces résultats s'expliquent par le fait que dans les conflits armés et les violences, les sujets jeunes, en particulier les hommes sont plus impliqués que les femmes et sont par conséquent plus exposés aux traumatismes.Les belligérants étaient touchés dans 39,3\% des cas suivis des élèves/étudiants $(21,4 \%)$. La fréquence élevée des belligérants que nous avons trouvée était également retrouvée par Thiam avec 36,9\%.Dans la série de 
Choua et al au Tchad en violence civile les élèves et étudiants $(39,5 \%)$ avaient dominé suivis des cultivateurs $(14,5 \%)$ et les sans emploi $(14,5 \%)$ (Thiam, 2018 ; Choua, 2016). La majorité des blessés (91\%) de cette étude provenaient de la capitale Bangui. Ce taux concordait avec la série de Choua dont $76 \%$ des blessés venaient de la capitale N'Djaména (Choua, 2016). Nous pouvons l'expliquer dans notre contexte par le fait qu'au mois de décembre 2013, les affrontements s'étaient concentrés dans la capitale Bangui ; ce qui avait fait plus de la moitié des blessés. Les circonstances des plaies chez nos patients étaient : les affrontements entre les belligérants (48,2\%), les agressions et rixes $(30,4 \%)$ et les balles perdues $(21,4 \%)$.Dans la série de Bahebeck les plaies relevaient d'une agression chez $56,5 \%$ des patients, un accident de chasse chez $20 \%$ et une faute de manipulation chez $2 \%$ des patents (Bahebeck, 2005). Pour Monneuse, 12 patients étaient blessés par autolyse, 10 par agression, 2 par accident de chasse, 2 en nettoyant leur arme et chez 6 patients la cause n'était pas précisée (Monneuse, 2004). Dans notre contexte de conflit armé, si les belligérants étaient par évidence blessés au cours des affrontements, les autres patients l'étaient accidentellement dans leur fuite pour se mettre à l'abri des tirs ou dans leurs déplacements dans le cadre de leurs activités habituelles ; voire même chez eux à domicile. La période du conflit avait entrainé des effets collatéraux tels que le changement de comportement des populations, la prise des stupéfiants et la pauvreté. Ce qui était à l'origine de l'augmentation de la criminalité et des violences d'où les agressions à mains armées de certains de nos blessés. Ce qui était confirmé par Ohene-Yeboah et al qui avaient rapporté que la prolifération des armes légères qui suivait les guerres civiles, les changements internes armés des processus politique savaient augmenté le taux de criminalité et ainsi, l'incidence des plaies pénétrantes de l'abdomen (Ohene-Yeboah, 2010).Les plaies provenaient d'une balle chez $84 \%$ des patients et des éclats de grenades chez $16 \%$ des patients dans l'étude. Mata Ambangne et al avaient retrouvé dans leur série que les éclats d'obus étaient en cause dans 73,5\% et les balles dans 26,5\% des cas (Mata Ambangene, 2013). Nous pouvons l'expliquer par le fait que chez les patients de notre étude, les hostilités avaient opposé des groupes armés non conventionnels contrairement aux affrontements interarmées d'où l'usage disproportionné des armes. Les véhicules privés $(44,6 \%)$, les ambulances non médicalisées $(35,7 \%)$, les motos-taxis $(12,5 \%)$ étaient les moyens les plus utilisés pour de transport des blessés. Ces blessés n'avaient reçu aucun soin initial jusqu'à l'admission. Dans les pays en développement comme le nôtre, l'absence de moyens d'évacuation adaptés dans nos structures de santé obligeait le transport des blessés avec tous les moyens disponibles ; ce qui était le cas chez les blessés de notre série comme rapporté dans la littérature (Ayité, 1996 ; Choua, 2016). La plupart des blessés $(71,4 \%)$ étaient admis aux urgences chirurgicales entre 50 minutes et 6 heures après le traumatisme contre 10,7\% 
au-delà de 24 heures ; le délai moyen était de 2,5 heures (extrêmes 50 minutes et 72 heures). Ce délai moyen, bien qu'inférieur à celui retrouvé par Choua qui était de 3,7 heures nous paraissait très long pour des patients blessés en majorité dans la capitale où se trouve le service d'accueil et s'expliquerait par les difficultés d'accès aux blessés à cause de l'insécurité (Choua, 2016).

\section{Aspects clinique et paraclinique}

Les plaies concernaient la région antérieure de l'abdomen dans 89,3\% des cas. Ce taux corrobore la série de Mata Ambangene et al au Congo Démocratique qui avaient trouvé 82,3\% (Mata Ambangene, 2013). Les sièges fréquents des plaies étaient : les flancs $(23,2 \%)$, les hypochondres $(21,4 \%)$, la région périombilicale $(16 \%)$ et les plaies thoracoabdominales $(12,5 \%)$.Ceci s'explique par la grande surface de la région antérieure de l'abdomen qui l'expose aux traumatismes comme le confirme la littérature (Ayité, 1996 ; Maha Yassin, 2014 ; Mata Ambagene, 2013). Par ailleurs dans notre contexte, l'atteinte de la région antérieure de l'abdomen et de ses diverses parties était due aux affrontements entre belligérants, aux blessés surpris par leurs agresseurs cachés ou aux balles perdues. Les blessés de la région postérieure l'étaient au cours de leur fuite et ceux des plaies thoracoabdominales par leurs agresseurs en altitude cachés dans des arbres ou les toits des maisons ou encore les balles perdues. La plupart des patients $(78,6 \%)$ avaient une plaie unique. Monneuse avait fait le même constat mais dans sa série dans des proportions différentes : 84,37\% de plaie unique, 15,63\% plaies multiples (Monneuse, 2004). La douleur abdominale était présente chez tous les patients. Les autres signes cliniques variaient selon les patients et étaient parfois associés. Dans la littérature, ces signes cliniques étaient retrouvés dans la plupart des séries (Bahebeck, 2005 ; Asuquo, 2012 ; Maha Yassin, 2014. (Mata Ambagene, 2013). La variation des signes cliniques dépendait de l'organe lésé et de l'importance de son atteinte; les lésions des organes pleins étant plus à l'origine d'un état de choc hypovolémique que les organes creux qui entrainent les signes cliniques de péritonite. Le groupe sanguin/facteur rhésus et le taux d'hématocrite et/ou le taux d'hémoglobine étaient les seuls examens biologiques réalisés chez les patients dans le cadre d'un bilan préopératoire. La radiographie de l'abdomen sans préparation était réalisée chez deux patients suspects d'une plaie thoracoabdominale dont l'abdomen était souple en dehors d'une douleur de l'hypochondre droit. Elle avait mis en évidence une rétention de la balle dans le foie.

\section{Aspect lésionnel}

La laparotomie était pratiquée chez 51 patients (91\%) sur les 56 ; les 5 autres blessés (9\%) avait fait l'objet d'une abstention chirurgicale. Il s'agissait de deux autres patients qui avaient présenté une épiplocèle par éclat de grenade 
et qui étaient hémodynamiquement stables sans signe d'irritation péritonéale. Leurs plaies étaient parées avec résection de l'épiploon sous anesthésie locale. Le troisième avait une effraction minime du péritoine sans éviscération ; la paroi était parée également sous anesthésie locale. Ils étaient cliniquement surveillés et n'avaient présenté aucune complication. Bahebeck et Maha Yassin avaient adopté cette attitude sélective d'abstention chirurgicale dans respectivement $12,8 \%$ et $18,8 \%$ de leurs séries en raison de l'absence de signes d'appel d'une indication opératoire (Bahebeck, 2005 ; Maha Yassin, 2014). Par contre, Ayité et Monneuse avaient adopté l'attitude de la laparotomie systématique en présence de toute plaie par arme à feu dans leurs séries en la justifiant par les fréquentes lésions organiques qu'entrainaient les projectiles en prenant le risque d'une laparotomie blanche (Ayité, 1996 ; Monneuse, 2004). Les deux autres patients avaient présenté une plaie thoracoabdominale avec une rétention de la balle dans le foie, stables avec un abdomen souple. Cette attitude non opératoire était confirmée par Starling et al qui avaient expliqué que dans une plaie thoracoabdominale avec rétention du projectile dans le foie, la probabilité d'un succès de prise en charge non opératoire était très grande (Starling, 2012). La réanimation préopératoire était réalisée chez 21 patients $(41,1 \%)$ dont 13 étaient reçus en état de choc hypovolémique d'emblée. Dans notre série, des lésions organiques étaient retrouvées chez 45 patients $(88,2 \%)$ à la laparotomie tandis que chez 6 patients $(11,8 \%)$, cette laparotomie était blanche. Ces résultats corroboraient la série de Bahebeck qui avait enregistré $22,5 \%$ de laparotomie blanche et Salim et al (Salim, 2002) qui avait rapporté que la laparotomie systématique dans les plaies abdominales par arme à feu entrainait 15 à $27 \%$ de laparotomie blanche (Bahebeck, 2005; Salim, 2002). C'est dire l'importance de moyens diagnostiques complémentaires qui font défaut dans nos pays en développement sous équipés. En peropératoire, 97 plaies organiques étaient répertoriées, dominées par les plaies du grêle (35\%) suivies de celles du colon $(23,7 \%)$ et du foie $(9,2 \%)$. Dans la littérature, le grêle, le colon et le foie sont les plus organes atteints dans les traumatismes ouverts (Bahebeck, 2005 ; Saidi, 2002 ; Maha Yassin, 2014 ; Mata Ambagene, 2013 ; Zeljko, 2004 ; Baradaran, 2007). Ceci se justifie d'une part par l'importance de leurs dimensions et de leur volume et d'autre part, par leur situation anatomique sous-pariétale qui les rend plus vulnérables. Quant aux plaies diaphragmatiques $(7,2 \%)$, elles relevaient de l'association des plaies thoracoabdominales comme certains auteurs les avaient retrouvées dans leurs séries (Bahebeck, 2005 ; Mata Ambagene, 2013 ; Starling, 2012). Des associations lésionnelles organiques allant de l'atteinte de 2 à 4 organes étaient retrouvées chez 48,9\% des patients. Ces atteintes polyviscérales étaient conformes à la littérature (Monneuse, 2004 ; Bahebeck, 2005 ; Ayité, 1996 ; Choua, 2016 ; Mata Ambagene, 2013). Les armes à feu sont responsables le 
plus souvent de la lésion de plusieurs organes à la fois contrairement aux armes blanches.

\section{Aspect thérapeutique}

Au plan thérapeutique, les gestes les plus pratiqués étaient l'excisionsuture des plaies des organes creux (grêle, colon, estomac, duodénum, vessie) et la suture des plaies des organes pleins (foie, pancréas, rein), 25 fois. L'iléostomie et la colostomie étaient réalisées, 21 fois et la résectionanastomose iléale et colique 17 fois. Les résections-anastomoses et les sutures des organes creux étaient systématiquement drainées après un lavage abondant au sérum physiologique de la cavité péritonéale. Ces gestes chirurgicaux conventionnels étaient retrouvés dans la littérature (Saidi, 2002 ; Salim, 2002 ; Monneuse, 2004 ; Arveux, 2007 ; Starling, 2012 ; Maha Yassin, 2014 ; Choua, 2016). Par contre, Bahebeck a rapporté que certains chirurgiens pratiquaient encore certaines méthodes jadis controversées comme la suture colique primitive sans lavage de la cavité péritonéale en la justifiant tantôt par le vide apparent du viscère, tantôt par le siège proximal de la plaie et se sont compliquées de suppuration ou de décès (Bahebeck, 2005). En effet, le lavage systématique de la cavité péritonéale, en particulier dans les lésions des organes creux (grêle, colon), permettait de réduire le risque de suppuration intrapéritonéale et pour le drainage, outre l'évacuation des sérosités, en cas de lâchage des sutures, il permettait d'obtenir des fistules dirigées contrairement aux fistules exposées responsables des péritonites postopératoires potentiellement plus septiques et plus graves. La morbidité était de $40 \%$ dans cette étude. Dans les séries de Monneuse et Bahebeck, elle était 34\% et 4\% (Monneuse, 2004 ; Bahebeck, 2005). Cette morbidité était dominée par la suppuration pariétale (56\%).Ces complications étaient retrouvées dans les interventions des organes creux tandis que le choc hémorragique provenait au décours des interventions sur le foie. Ces complications étaient conformes à la littérature (Ayité, 1996 ; Adesanya, 1998, Asuquo, 2012 ; Choua, 2016 ; Salim, 2002). Le taux élevé des infections postopératoires retrouvé pourrait se justifier par la promiscuité des patients ainsi que de leurs familles du fait de la faible capacité du service qui était le seul à fonctionner au plus fort de la crise pendant les mois d'Octobre et Décembre 2013 et au cours desquels nous avons enregistré le plus grand nombre de blessés. Ces patients étaient hospitalisés dans des chambres surchargées ou sous des tentes. A cela s'ajoutaient l'absence de prise en charge initiale sur les lieux des traumatismes et en cours des évacuations ainsi que la qualité des soins postopératoires et l'insuffisance ou le manque de sang. Dix patients sur les 56 étaient décédés soit un taux de mortalité de 17,9\%. Dans la littérature, ce taux variait entre 5,5\% et 18\% (Monneuse, 2004 ; Bahebeck, 2005 ; Ayité, 1996 ; Salim, 2002). Les causes des décès étaient le choc hémorragique, la septicémie et la 
péritonite postopératoire dans respectivement cinq, trois et deux cas. Les complications hémorragiques et infectieuses des plaies organiques responsables des décès dans la série étaient retrouvées par la plupart des auteurs (Monneuse, 2004 ; Bahebeck, 2005 ; Asuquo, 2012 ; Choua, 2016). La durée moyenne de séjour hospitalier était de 10 jours extrêmes (1 jour et 54 jours). Elle était de 10,5jours dans la série de Monneuse (extrêmes 1 et 83 jours (Monneuse, 2004). Elle s'expliquerait dans cette étude par la faible capacité d'accueil du service et surtout en cette période de conflit où il y avait un afflux de blessés qui nous obligeait à faire des sorties précoces dès que leur état clinique le permettait.

\section{Conclusion}

Les plaies abdominales par armes à feu sont une urgence chirurgicale digestive absolue en raison des lésions pour la plupart pluriviscérales qui engagent le pronostic vitale. Elles touchent les sujets jeunes, actifs dans les conflits armés et les violences. La prise en charge initiale des patients dans notre contexte était inexistante en préhospitalier et insuffisante en milieu hospitalier à l'origine d'une morbi-mortalité élevée. L'organisation des services de santé et leur équipement permettant la prise en charge des blessés depuis les lieux de ramassage, pourraient réduire ces complications.

Tableau I : Répartition selon les tranches d'âge des plaies pénétrantes de l'abdomen par armes à feu des patients admis au CHU Communautaire de Bangui, Centrafrique $(n=56)$

\begin{tabular}{lcc}
\hline Tranches d'âge (ans) & Effectif & Pourcentage \\
\hline $11-20$ & 10 & 17,8 \\
$21-30$ & 29 & 51,8 \\
$31-40$ & 8 & 14,3 \\
$41-50$ & 4 & 7,1 \\
$51-60$ & 4 & 7,1 \\
Plus de 60 & 1 & 1,8 \\
Total & 56 & 100 \\
\hline
\end{tabular}

Tableau II : Répartition selon le siège des plaies pénétrantes de l'abdomen par armes à feu des patients admis au CHU Communautaire de Bangui, Centrafrique ( $\mathrm{n}=56$ )

\begin{tabular}{ccc}
\hline Siège des plaies & $\begin{array}{l}\text { Effectifs } \\
(\mathbf{n})\end{array}$ & $\begin{array}{l}\text { Pourcentage } \\
(\mathbf{\%})\end{array}$ \\
\hline Flancs & 11 & 19,6 \\
Périombilical & 9 & 16,1 \\
Fosses iliaques & 5 & 8,9 \\
Thoracoabdominal & 7 & 12,5 \\
Hypochondres & 12 & 21,4 \\
Hypogastre & 3 & 5,4 \\
Epigastre & 3 & 5,4 \\
Région dorsale & 6 & 10,7 \\
Total & $\mathbf{5 6}$ & $\mathbf{1 0 0}$ \\
\hline
\end{tabular}


Tableau III : Répartition selon les gestes chirurgicaux pratiqués des plaies pénétrantes de l'abdomen par armes à feu des patients admis au CHU Communautaire de Bangui, Centrafrique

\begin{tabular}{ccr}
\hline Organe lésé & Geste pratiqué & Nombre \\
\hline Grêle & Résection-anastomose & 12 \\
Colon & & 5 \\
Grêle & Stomie & 12 \\
Colon & & 9 \\
Estomac & & 5 \\
Foie & & 6 \\
Grêle & Suture & 4 \\
Colon & & 2 \\
Mésentère & & 2 \\
Epiploon & & 2 \\
Pancréas & & 1 \\
Duodénum & & 1 \\
Vessie & & 1 \\
Rein & & 3 \\
Rate & & 1 \\
Vésicule biliaire & Ablation totale & 1 \\
Rein & & 2 \\
Foie & Ablation partielle & 1 \\
Epiploon & Tamponnement (packing) & 2 \\
Foie &
\end{tabular}

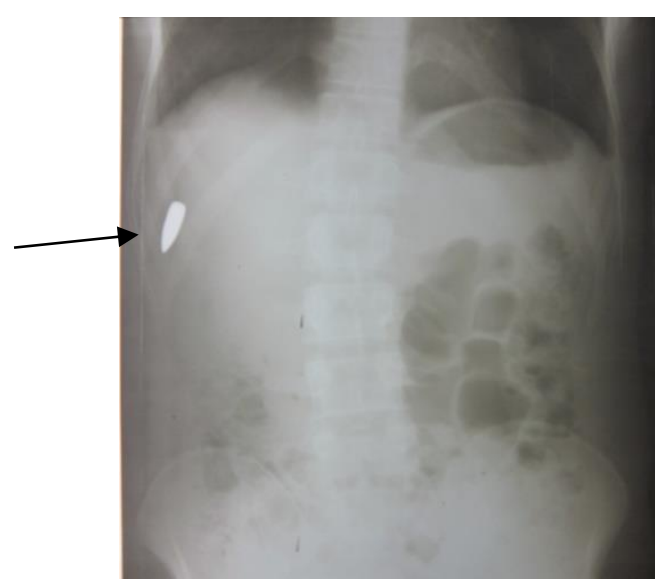

ASP de face

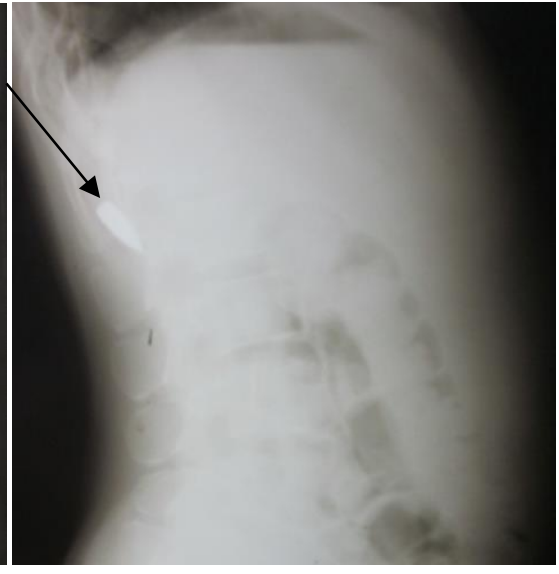

ASP profil

Figure 1 : Rétention d'une balle dans le foie chez un patient de 20 ans victime d'une plaie thoracoabdominale à porte d'entrée sus claviculaire droite.

\section{Références :}

1. Adesanya AA, Afolabi JR da Rocha, Afodu JT, et al (1998). Civilian abdominal gunshot wounds in Lagos. J R Coll Surg Edinb, 43 (4): 230234. 
2. Asuquo M, Umoh M, Nwagbara V,et al (2012). Penetrating abdominal trauma: experience in a teaching hospital, Calabar, Southern Nigeria. Intern J Clin Med, 3 (5) : 456-430.

3. Arveux C, Letoublon C, Reche F (2007). Le damag control en traumatologie abdominale sévère. Réanimation, 16 : 678-686.

4. Ayité A, Etey K, Fekete L, et al (1996). Les plaies pénétrantes de l'abdomen au CHU de Lomé. A propos de 44 cas. Med Afr Noire, 43 (12) : 642-46.

5. Bahebeck J, Masso Missé, Essomba A, et al (2005). La plaie abdominale par balle : à propos de 86 au Cameroun. Med Trop, 65 (6) : 554-558.

6. Baradaran H, Salimi J, Nassaji-Zavareh M, et al (2007). Epidemiological study of patients with penetrating abdominal trauma in Tehran-Iran. Acta Medica Iranica, 45 (4) : 305-308.

7. Bodalal Z, Monsor S (2013). Gunshot injuries in Benghazi-Libya in 2011 : the Libyan conflit and beyong. US Library of Medecine, 11 (5) : 258-63.

8. Choua O, Rimtebaye K, Adam Adami M, et al (2016). Les plaies pénétrantes par armes blanches et à feu à N'djamena, Tchad : une épidémie silencieuse ? Eur Scient J, 12 (9) : 180-191.

9. Dieng M, Wilson E, Konaté I, et al (2003). Plaies pénétrantes de l'abdomen : «abstentionnisme sélectif» versus laparotomie systématique. e-mémoires de l'Académie Nationale de Chirurgie, 2 (2) : 22-25.

10. Maha Yassin O, Aamir Abdullahi H, Mohammed Toum M (2014). Penetrating abdominal injuries : Pattern and outcome of management in Khartoum. Int J Clin Med, 5 : 18-22. 12.

11. Mata Ambangene D, Wami w'Ifongo F (2013). Plaies abdominales et pelviennes pénétrantes par armes à feu à Kisangani en en province orientale, Congo. Aspects anatomopathologiques. Rev méd Gd Lacs, 2 (2) : 173-185.

12. Mnguni MN, Muckart DJJ, Madiba TE (2012). Abdominal trauma in Durban, South Africa : Factors infuencing outcome. Int Surg, 97 : 161168.

13. Monneuse OJ, Barth X, Gruner L, et al (2004). Les plaies pénétrantes de l'abdomen, conduite diagnostique et thérapeutique. A propos de 79 patients. Ann Chir, 129 (3) :156-163

14. Moore EE, Marx JA, Dakubo JCB, et al (1985). Penetrating abdominal wound. Rational for exploratory laparotomy. JAMA, 258 : 2708-2711.

15. Ohene-Yeboah M, Dakubo JCB, Boakye F, et al (2010). Penetrating abdominal injuries in adults seen at two teaching hospitals in Ghana. Ghana Med J, 44 (3) : 103-108. 
16. Saidi HS, Nyakiamo J, Faya S (2002). Gunshot injuries as seen at the Aga Khan hospital, Nairobi, Kenya. East Afr Med J, 79 (4) : 188-192.

17. Salim A, Velmahos GC (2002). When to operate on abdominal gunshot wounds. Scandinavian J Surg, 91 : 62-66.

18. Starling SV, Rodigues BL, Portes Rocha Martins M et al (2012). Non operative management of gunshot wounds on the right thoracoabdomen. Rev Col Bras Cir, 39 (4) :286-294.

19. Thiam S, Diakité M, Mallé M, et al (2018). Epidémiologie des plaies par balle et engins explosifs à Gao (Mali) : Série de 303 cas. Jaccr Africa, 2 (3) : 354-360.

20. Zeljko B, Zvonimir L, Enio A, et al (2004). Small bowel injuries in penetrating abdomminal trauma during war: ten-year follow-up findings. Military Medecine, 169 (9) : 721-722. 